\title{
Towards a Taxonomy of Wired and Wireless Anonymous Networks
}

\author{
Douglas Kelly, Richard Raines, Rusty Baldwin, Barry Mullins, and Michael Grimaila \\ Air Force Institute of Technology \\ Wright Patterson AFB OH 45433 \\ \{Firstname.Lastname\}@afit.edu
}

\begin{abstract}
With the aim to preserve privacy over a communications network, a plethora of anonymous protocols have been proposed along with many empirical investigations into specific adversary attacks over those networks. However, no known taxonomies exist that address anonymity in the diverse set of both wired and wireless anonymous communications networks. This paper proposes such a novel cubic taxonomy which explores the three key components of anonymity property, adversary capability, and network type. This taxonomy expands the definition of anonymity and aids in the advancement of state-of-the-art technological privacypreserving mechanisms in anonymous networks against any adversary.
\end{abstract}

Index Terms-Anonymity, privacy, taxonomy, communications networks

\section{INTRODUCTION}

A s millions of on-line users become subject to increasing eavesdropping, profiling, and tracking, privacy is becoming paramount. Over the past two and one-half decades, the vast majority of anonymity research has been on proposing anonymous communications networks and analyzing traffic over these networks. For example, numerous networking protocols designed to achieve anonymity include Anonymizer [1], Crowds [2], Tarzan [3], and Herbivore [4] for web surfing; Mixminion [5] for e-mail; Freedom Network [6], $\mathrm{P}^{5}$ [7], and Tor [8] for interactive web and relay chat; Secure Dynamic Distributed Routing (SDDR) [9], Anonymous On-Demand Routing (ANODR) [10], and MASK [11] for hostile wireless military environments; authentication protocols [12] for WiMAX networks; Homeless Protocol [13], enhanced Third-Generation Partner Project (3GPP) Anonymity Preserving Authentication and Key Agreement (AP-AKA) protocol [14], and other authentication protocols $[15,16]$ for cellular networks; Anonymous and Authenticated Key Exchange for Roaming networks (AAKE-R) [17] such as inter-bank ATM networks and credit card payments systems; Simple Anonymity Scheme (SAS) [18] for clustered wireless sensor networks; and Mutual Anonymous Multicast (MAM)

All authors are with the Air Force Institute of Technology, WPAFB, OH 45433 USA (corresponding author phone: 502-542-1646; fax: 937-656-7342; e-mail: douglas.kelly@afit.edu).
[19], Bus-based Anonymous Multicast [20] (BAM), and Anonymous Symmetrically Cryptographic (ASC) [21] for real-time audio and video conferencing. The privacypreserving technologies of these and other protocols offer varying types and levels of anonymity protection over a communications network. Their effectiveness depends heavily on the number of anonymous users, how messages are routed, adversary knowledge and ability, and other Internet $[22,23]$ and/or mobile network $[24,25]$ environmental factors. Hence, an ability to formally compare these anonymity protocols and anonymity services is imperative.

This motivates the development of a novel cubic taxonomy (CT) to facilitate the systematic definition and comprehensive classification of anonymity in existing and proposed wired and wireless anonymous communications networks. The CT approach accentuates the significance and subtlety of anonymity and acts as a state-of-the-art taxonomy for ongoing anonymity research.

The rest of the paper is organized as follows. Section II describes related anonymity research and how CT augments such work. Section III introduces the novel CT. Section IV defines the anonymity property component. Section V delineates the adversary capability component. Section VI details the network type component. Section VII demonstrates the utility of CT by classifying anonymous networks in 3D cubic and 2D taxonomies and summaries the advantages they offer. Section VIII concludes the paper.

\section{RELATED WORK}

In an attempt to categorize anonymous systems, three known anonymity-related conceptual taxonomies have been proposed: a group support system (GSS) taxonomy [26], a lower-level collaborative peer group (CPG) taxonomy [27], and a connection anonymity taxonomy (CAT) [28]. In the GSS, the four components are anonymity factors, anonymity types, individual anonymity and group process/outcome with arrows indicating a natural flow from the anonymity factors to the eventual group outcome. Unlike GSS, our CT extends the definition of anonymity beyond simply individual sender and receiver anonymity and takes into account the crucial adversary capability and anonymous network type components. In the CPG, the peer-to-peer network has identity-less peers and template-based peer groups designed to facilitate the development and deployment of applications where self-organizing peers aggregate in a controlled manner and new types of communication primitives achieve collective 
goals. Like CPG, our CT incorporates the potentially peer and group identity-less aspects of anonymity properties and network routing strategies. However, our CT is a high-level conceptual taxonomy, not a low-level middleware architecture and software toolkit, and is useful for considering anonymity in more than just low density ad-hoc mobile networks. The CAT consists of design factors, connection anonymity functions, and objectives components and is a structured metalevel approach to designing and comparing current anonymity strategies and techniques. Although developed independently, $\mathrm{CT}$ extends the CAT taxonomy. The CT and CAT threat model components are nearly synonymous albeit CT more abstractly and precisely defines certain terms. Our CT expands the CAT routing strategies classification. This further refines the protocol family relationships. Unlike CAT, CT considers a more inclusive and subtle definition of anonymity. Also, CT results in a visually intuitive $3 \mathrm{D}$ or $2 \mathrm{D}$ comparison of anonymous networks.

In addition to other taxonomies, various surveys or tutorials [29-31] depict other interesting but more focused aspects of anonymity such as location anonymity in wireless networks, classification of mixnets, and supplementary term definitions [32, 33]. Fortunately, the CT definition of anonymity and classification of network types is inclusive enough to capture these proposed future anonymous protocols and effortlessly compare them with existing anonymous protocols. Another survey classified a subset of anonymous wired networks [34], namely mixnets. Unlike CT, the classification is by mixnet type and verifiability mechanism which works especially well for cascade mixnets. However, CT offers a complementary classification for peer-to-peer anonymous network solutions as well as mixnets. Also, no other known surveys or tutorials have offered a synthesized classification of both wired and wireless anonymous protocols. Finally, proposed anonymous network protocols often cite their relative advantages and disadvantages in contrast to other protocols. For instance, Hierarchical Anonymous On-demand Routing (HANOR) [35] is compared with five other protocols, WonGoo [36] with Crowds and mixnets, AnonDSR [37] with SDAR [38], and MASK with ANODR. Hence, proposed protocols are compared with few other anonymous protocols. CT allows the straightforward classification and comparison of an arbitrary number of anonymous networks into protocol families.

\section{CUBic TAXONOMY}

A novel three-dimensional (3D) cubic taxonomy is proposed to classify the desired anonymity properties, presumed adversary capabilities and selected network types inherent in an anonymous communications network. This cubic taxonomy (CT) is shown in Figure 1.

The top-level contains three fundamental components: Anonymity Property, Adversary Capability, and Network Type. Anonymity Property addresses "What information must be hidden?" Hiding identity, relationship, location and/or other items of interest (IOI) from others in the anonymous network is typical. Adversary Capability addresses "From whom do we hide it?" It defines who the assumed adversary is and how strong the threat to the anonymous system is.

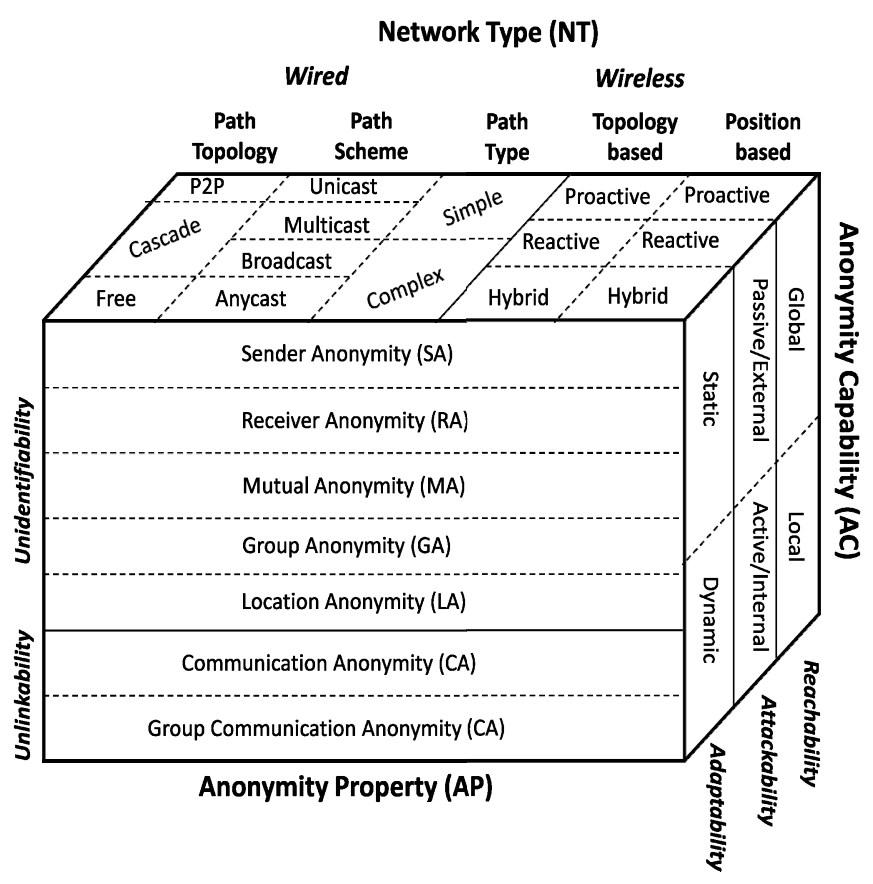

Figure 1: Cubic Taxonomy (CT) Components

Network Type addresses "How hidden must it be?" by defining routing schemes, the transmission medium, network topology, and protocol interdependencies impact on anonymity. These three components are further decomposed as shown in Figure 1.

At this mid-level, the Anonymity Property is broken down into the abstract unidentifiability and unlinkability terms. The Adversary Capabilities are broadly categorized as reachability, attackability, and adaptability. Finally, Network Type is either wired or wireless. These seven sub-components are further decomposed into their twenty-eight (28) smallest subcomponents.

The bottom-level consists of seven anonymity properties, six adversary capabilities and five network types decomposable into fifteen network sub-strategies. This is the first known 3D synthesized graphical classification of both wired and wireless anonymous networks.

\section{ANONYMITY PROPERTY}

Anonymity properties are generally classified into unidentifiability, unlinkability, and unobservability; however, only the former two are included in this taxonomy since the latter automatically implies anonymity [39]. Unidentifiability means the adversary is unable to discern an agent's or group's identity, actions or other IOI among a similar set of agents or groups. Unlinkability means the adversary is unable to relate agents, messages, actions or other IOI by observing the system. Moreover, an adversary's a priori and a posteriori knowledge are the same even after observing the IOI. The classical definition of anonymity is

Anonymity $=$ Unidentifiability + Unlinkability. 
TABLE I

ANONYMITY PROPERTIES

\begin{tabular}{cll}
\hline \hline Property & \multicolumn{1}{c}{ Type } & \multicolumn{1}{c}{ Hidden Information } \\
\hline Unidentifiability & Sender Anonymity (SA) & Message sender identity \\
& Receiver Anonymity (RA) & Message receiver identity \\
& Mutual Anonymity (MA) & Message identities from each other \\
& Group Anonymity (GA) & Message group identity \\
& Location Anonymity (LA) & Position, motion, link, or topology information \\
Unlinkability & Communication Anonymity (CA) & Sender-Receiver pair relationship from others \\
& Group Communication Anonymity (GCA) & Group-Group pair relationship from others \\
\hline \hline
\end{tabular}

\section{A. Unidentifiability}

Unidentifiability is decomposed into sender anonymity (SA), receiver anonymity (RA), mutual anonymity (MA), group anonymity (GA), and location anonymity (LA) [39]. SA prevents a particular message from being linked to a particular sender identity. The Dining Cryptographers Network (DC-Net) [40] mechanism achieves sender anonymity. RA prevents a particular message from being linked to a particular receiver identity. Broadcast $[41,42]$ and private information retrieval [43] are two mechanisms that achieve receiver anonymity. MA hides the sender and receiver identities from each other. GA limits the adversary to linking a particular message to a group of agents. Agent identity is hidden among a group of indistinguishable agents. At a higher level of abstraction, group anonymity prevents a particular message from being linked to a particular group of agents. However, no known group anonymous services exist yet. The MAM aims to achieve both mutual and group anonymity. LA means a particular message is not linkable to any sender or receiver location, motion, route or topology information. Many wireless anonymous protocol approaches $[10,11,21,29,30,44-49]$ aim to achieve location anonymity. The classic, current, and extended cubic unidentifiability property definitions are

$$
\begin{aligned}
& \text { Classic Unidentifiability }=\mathrm{SA}+\mathrm{RA} \\
& \text { Current Unidentifiability }=\text { Classic Unidentifiability }+\mathrm{LA} \\
& \text { Cubic Unidentifiability }=\text { Current Unidentifiability }+\mathrm{MA}+\mathrm{GA}
\end{aligned}
$$

\section{B. Unlinkability}

Unlinkability consists of communication anonymity (CA) and group communication anonymity (GCA). CA means a particular message cannot be linked to any sender-receiver pair and no message is linkable to a particular sender-receiver pair. The MIX-net [50] mechanism achieves CA. CA is a weaker property than each of sender and receiver anonymity. GCA means a particular message cannot be linked to any sender group-receiver group pair and no message is linkable to a particular group sender-group receiver pair. All known anonymity research on the unlinkability property primarily deals with CA. The classic and extended cubic unlinkability property definitions are
Cubic Unlinkability = Classic Unlinkability + GCA

Given these first two anonymity properties, the classic and expanded anonymity definitions are

$$
\begin{aligned}
\begin{aligned}
\text { Classic Anonymity } & =\text { Classic Unidentifiability }+ \text { Classic Unlinkability } \\
& =\mathrm{SA}+\mathrm{RA}+\mathrm{CA}
\end{aligned} \\
\begin{aligned}
\text { Expanded Anonymity } & =\text { Cubic Unidentifiability }+ \text { Cubic Unlinkability } \\
& - \text { Classic Anonymity } \\
& =\mathrm{LA}+\mathrm{MA}+\mathrm{GA}+\mathrm{GCA}
\end{aligned}
\end{aligned}
$$

Finally, the new cubic anonymity definition is

$$
\begin{array}{r}
\text { Cubic Anonymity }=\text { Cubic Unidentifiability }+ \text { Cubic Unlinkability } \\
\text { OR } \\
=\text { Classic Anonymity }+ \text { Expanded Anonymity }
\end{array}
$$

\section{ADVERSARY CAPABILITY}

An adversary is an agent or set of agents whose aim is to degrade or eliminate anonymity. This is normally an untrusted third party; however, in roaming networks such a cellular networks, the adversary may also be a legitimate network agent such as the home or foreign server agent. The adversary capabilities range from weak to strong and represent the assumed threat model. Table II lists capabilities, their type and a brief description. The next sections explain each capability further.

\section{A. Reachability}

Reachability is either global or local. A global adversary is omnipresent and has full access to the entire network of nodes and links. A local adversary has limited omnipresence and has full access to only a portion of the network nodes and links. This corresponds to the adversary possessing complete

TABLE II

ADVERSARY CAPABILITIES

\begin{tabular}{cll}
\hline \hline Capability & \multicolumn{1}{c}{ Type } & \multicolumn{1}{c}{ Description } \\
\hline Reachability & Global & Omnipresent \\
& Local & Limited omnipresent \\
Attackability & Passive/External & Compromise links \\
& Active/Internal & Compromise nodes \\
Adaptability & Static & A priori knowledge \\
& Dynamic & Posterior knowledge \\
\hline \hline
\end{tabular}


or restricted information or knowledge about the system. It may also refer to the veracity of this information. The adversary may either know things to be true or only believe things to be true.

\section{B. Attackability}

Attackability is the combination of passive/external or active/internal. The objective of any attack is to link sender and receiver, identify the sender or receiver for a particular message, trace a sender forward/receiver back to messages or disrupt the system.

A passive/external adversary is an outsider that can only observe messages traversing the network and is typically invisible. This adversary can only compromise communication channels between nodes. In other words, it is a non-empty set of agents, part of the surrounding of the anonymous system and capable of compromising links.

An active/internal adversary is an insider and may alter messages traversing the network but is visible. This adversary controls nodes in the network. In other words, this describes a non-empty set of agents which are part of the anonymous system and capable of participating in normal communications and controlling at least some nodes.

\section{Adaptability}

Adaptability describes whether the adversary or the anonymous system is static or dynamic. Typically, the adversary is dynamic and collects information about the path selection algorithm, its parameters and as much information as possible about network activities from compromised nodes and links. The adversary uses all available facts to infer who sent or received which messages in a computationally bounded or even unbounded manner. The adversary may behave deterministically with a scheduled plan of attack, probabilistically depending on the relative frequency of sequences of observed actions or events, or nondeterministically (unpredictably). The adaptability of the anonymous system determines if or how much information is leaked to the adversary. A static system keeps the adversary knowledge about the network and agent targets constant during and after an attack. So the adversary retains only $a$ priori knowledge. A dynamic system may attempt to thwart an adversary's ongoing attack but may allow the adversary to learn additional information and update knowledge about the network and agent targets. So the adversary's a posterior knowledge may be greater than a priori knowledge. The network types are described next.

\section{NETWORK TYPE}

Anonymous networks exist as either wired or wireless. Anonymous communications networks typically vary in routing scheme, transmission medium, topology, and protocol implementation which affect the adversarial threat. Hence, providing anonymity in each network requires a different approach particularly when mobility is involved. Table III outlines each type, its subtypes, related routing, and a brief description. Wired anonymous networks are examined in the next section.

\section{A. Wired}

Wired networks may be decomposed into path topology, route scheme, and path type strategies. Each strategy assumes static a priori topology knowledge of the anonymous network for the duration of an adversary's attack.

The Path Topology routing approaches are cascade and free route for mixnets [34] or distributed for P2P networks. In a cascade network, senders choose from a set of fixed paths through the anonymous network for message transfer. In free route or P2P networks, senders may choose a route of variable length through the network for message transfer.

The Route Scheme is a major factor affecting anonymity. Four generic network routing schemes are shown in Figure 2. Practically all in-depth research on wired anonymity networks assumes a unicast routing strategy. Exceptions include the DC-Net, $\mathrm{P}^{5}$, Hordes [51], MAM, and Cashmere [52].

Two Path Type approaches are simple and complex. In a simple path, no cycles are allowed. Intermediate nodes may only appear once on the path. In a complex path, cycles are allowed. In one strategy, the cycles may be disjoint. These cycles share no common nodes. Only intermediate nodes at the starting and ending point of a cycle can appear exactly twice on the path. In another strategy, the cycles may be arbitrary. This path begins and ends with the same node but

TABLE III: NETWORK TYPES

\begin{tabular}{|c|c|c|c|}
\hline Type & Sub-type & Routing & Description \\
\hline \multirow[t]{9}{*}{ Wired } & Path Topology & Cascade & Fixed path length \\
\hline & & Free & Variable path length \\
\hline & & $\mathrm{P} 2 \mathrm{P}$ & Dynamic path length \\
\hline & Route Scheme & Unicast & One-to-one only \\
\hline & & Multicast & One-to-many \\
\hline & & Broadcast & One-to-all \\
\hline & & Anycast & $\begin{array}{l}\text { One-to-one among possible } \\
\text { many }\end{array}$ \\
\hline & Path Type & Simple & No cycles \\
\hline & & Complex & Allow cycles \\
\hline \multirow[t]{6}{*}{ Wireless } & Topology-based & Reactive & $\begin{array}{l}\text { Identity-based, on-demand, } \\
\text { high mobility }\end{array}$ \\
\hline & & Proactive & $\begin{array}{l}\text { Identity-based, table-based, } \\
\text { low mobility }\end{array}$ \\
\hline & & Hybrid & Combined reactive/proactive \\
\hline & Position-based & Reactive & $\begin{array}{l}\text { Identity-free, on-demand, } \\
\text { high mobility }\end{array}$ \\
\hline & & Proactive & $\begin{array}{l}\text { Identity-free, table-drives, } \\
\text { low mobility }\end{array}$ \\
\hline & & Hybrid & Combined reactive/proactive \\
\hline Hybrid & & & Combined wired/wireless \\
\hline
\end{tabular}

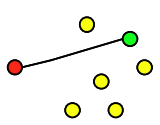

(a) Unicast

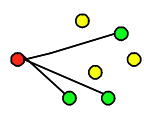

(b) Multicast

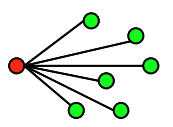

(c) Broadcast

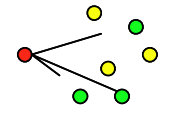

(d) Anycast
Figure 2: Network Routing Schemes 
intermediate nodes may appear an arbitrary number of times.

\section{B. Wireless}

The Wireless Network Type is decomposed into topologybased and position-based. Topology-based protocols use information about links in the network to perform packet forwarding. Position-based routing protocols use geographical node position information to make routing decisions. A mobile wireless node typically broadcasts to neighboring nodes so no route scheme is strictly necessary when classifying anonymous wireless networks. Either routing protocol may be classified as proactive, reactive, or hybrid. Proactive protocols periodically exchange control messages to make routing adaptations in the network. The control messages may be sent locally to discover neighbor nodes or more distributed to obtain topology information from all network nodes. Either way, a route is known in advance. Reactive protocols do not discover routes in advance but rather attempt to find routes on-demand and routes request packet across the network prior to sending any data. Hybrid or "zone" protocols use a mix of both proactive and reactive routing techniques at the network node. No one routing protocol is universally applicable.

\section{ANONYMOUS NETWORK TAXONOMIES}

The cubic taxonomy allows the protocol families classification of state-of-the-art anonymous networks. The utility of CT is demonstrated two ways. First, using the 3D cubic taxonomy, a select few anonymous protocols are compared with all three components. Second, using a 2D tree taxonomy, thirty-one anonymous protocols are examined via the Anonymity Property and Network Type components only. To the best of our knowledge, this is a more comprehensive classification of wired protocol family relationships and first known to capture wireless protocol family relationships. It is also the first graphical synthesized classification of both wired and wireless anonymous networks.

\section{A. 3D Cubic Taxonomy}

The purpose of CT is to visually compare different anonymous network protocols and group them into identifiable protocol families. The taxonomy is used to classify a variety of wired

For instance, DC-Net, Crowds [8, 53], and Tor [8, 54] anonymous networks are compared in Figure 5. For AP, each offers SA and RA against specific adversaries; in addition, Tor offers CA. For AC, DC-net assumes a strong passive global threat model whereas Crowds and Tor assume a weaker local adversary threat model. However, the latter two offer some degree of anonymity against an active, dynamic adversary who may control a limited number of collaborating jondos or compromised onion routers as well as selective passive traffic analysis attempts. For NT, all three are wired networks; however Tor employs a free route path topology whereas DCNet and Crowds are P2P. Also, DC-Net uses a broadcast route scheme whereas Crowds and Tor use unicast and allow complex path types. Hence, formally analyzing similar anonymous protocols such as Crowds and Tor which offer anonymous web-surfing may prove to be an intriguing investigation. However, if two protocols are conceptually very different such as DC-Net and Tor, then any comparison may prove to be difficult or simply invalid.

Also, the Secure Distributed Anonymous Routing (SDAR) [38] and Zone-based Anonymous Routing Protocol (ZAP) [55] anonymous network protocols are compared in Figure 4. In terms of NT, both are wireless networks; however ZAP is a hybrid, position-based protocol that uses destination flooding where as SDAR is a hybrid, topology-based protocol that uses multicast. In terms of AC, both assume a local, passive/external adversary; however, adaptability for ZAP may be static with a fixed receiver anonymous zone or dynamic with an adaptive receiver anonymous zone. Attackability may be active/internal for SDAR, but only passive/external for ZAP. In terms of AP, both offer SA and
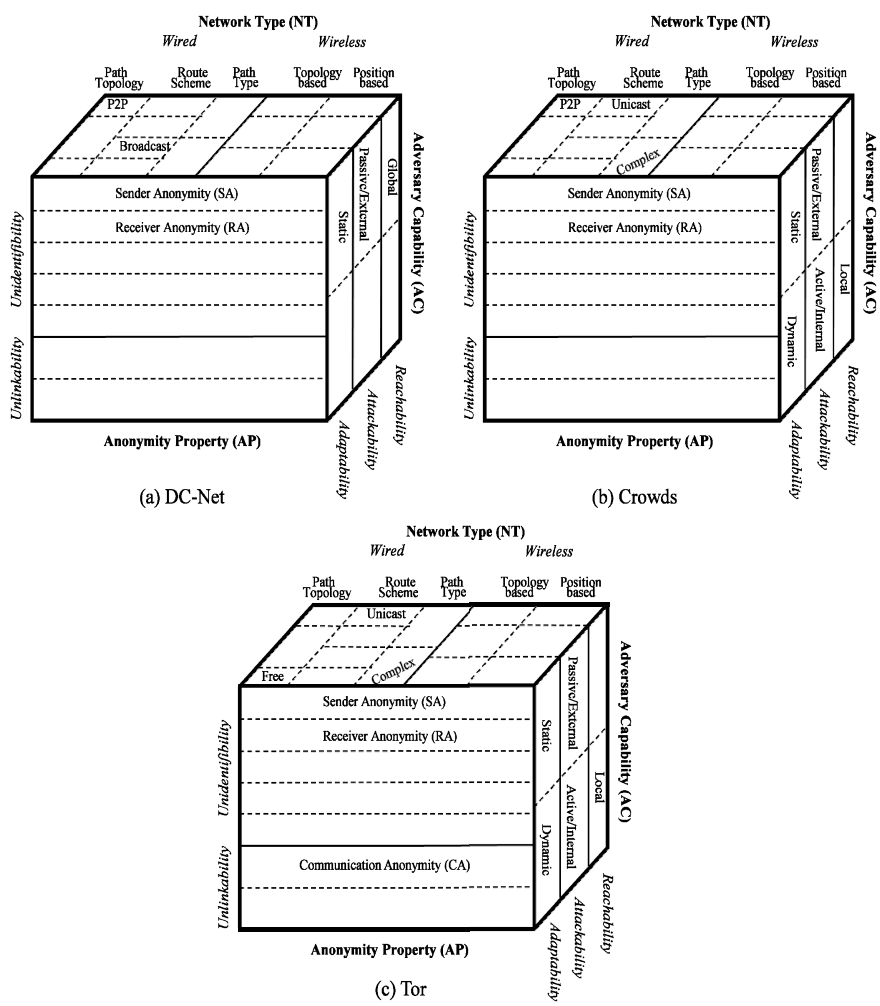

Figure 3: Cubic Taxonomy of Wired Anonymous Protocols
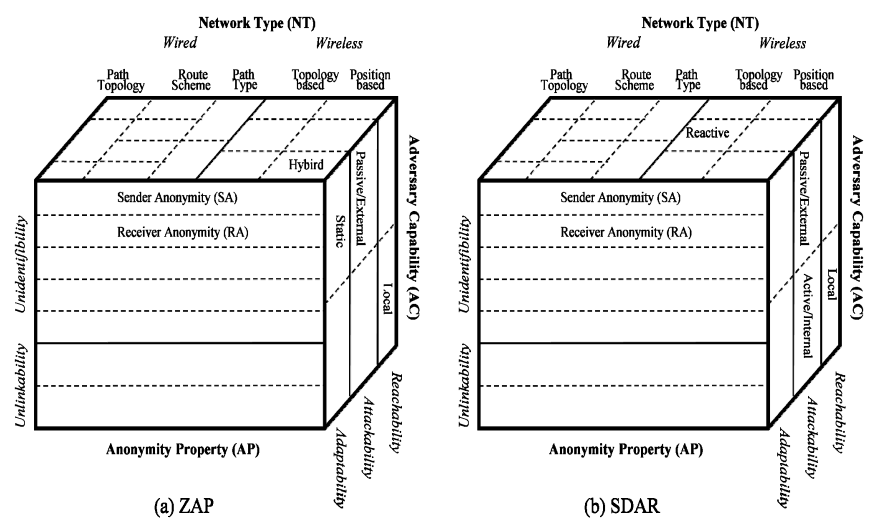

Figure 4: Cubic Taxonomy of Wireless Anonymous Protocols 
RA. Hence, formally representing these two protocols and/or quantitatively comparing their anonymity preservation and degradation may prove to be an intriguing investigation. In the end, a family of anonymous networking protocols may be more closely and rigorously analyzed.

\section{B. $2 D$ Tree Taxonomy}

The 2D tree-based taxonomy is shown in Figure 5. The internal tree structure from the Anonymous Network root node down to Protocol Name and Protocol Acronym nodes correspond to the Network Type classification displayed in Table III. The leaf nodes represent the Anonymity Types specified in column 2 of Table I.

The overall classification of twenty-one wired anonymous network protocols is shown in Figure 6. This taxonomy classifies classical and state-of-the-art wired anonymous networks. It adds path type and routing scheme classification and fills in the previously lacking P2P overall classification. JAP [56], Onion-Routing I [57], PipeNet [58], and Freedom Network [6] use cascade topologies. Onion-Routing II (Tor) [8], Cyberpunk [33], Mixmaster [59], and Mixminion [5] use free-route topologies. Tarzan [3], Crowds [53], WonGoo [36], Hordes, MAM, DC-net, $\mathrm{P}^{5}$, Herbivore, and Cashmere are P2P protocols. Herbivore uses a broadcast strategy whereas $\mathrm{P}^{5}$ employs a tree broadcast strategy. Hordes, M2 [60], AP3 [61], Secure Anonymous Multicast (SAM), BAM, and MAM use a multicast strategy. Only Cashmere uses an anycast strategy. All but Onion Routing II (Tor), Crowds and WonGoo use a simple path type strategy. Crowds and WonGoo allow a complex arbitrary cycle path type. PipeNet, Freedom, Crowds, and WonGoo offer sender anonymity only. Onion Routing II (Tor), Mixminion, Tarzan, and $\mathrm{P}^{5}$ offer classical anonymity of sender, receiver and communication anonymity. Herbivore does also if the receiver is inside the anonymous network. This 2D taxonomy is a valid classification of wired anonymous networks since Cyberpunk, Mixmaster, and Mixminion form a single protocol family under the

Anonymity

Network $\rightarrow$ Wired $\rightarrow$ FreeRoute $\rightarrow$ Unicast $\rightarrow$ Simple

classification. This matches the recent and complementary Anonymity $\rightarrow$ Mixnet $\rightarrow$ Freeroute $\rightarrow$ Asynchronous $\rightarrow$ Remailer classification [34]. However, this new taxonomy classifies more wired networks such as Cashmere, MAM and WonGoo and classifies P2P networks in addition to classical mixnets.

This is the first known capture of wireless anonymous networks into protocol families. SDR, Anonymous Dynamic Source Routing (AnonDSR) [37], Anonymous Routing for Mobile Ad hoc networks (ARM) [62], On-Demand Anonymous Routing (ODAR) [48], HANOR, and MASK [11] are topology-based protocols. ANODR, SDDR, ASR [44], Anonymous On-Demand Position-based Routing (AODPR) [63], Ad hoc On-demand Position-based Private Routing (AO2P) [49], SAS, ASC, and ZAP [55] are position-based protocols. SDAR, MASK and ZAP use the hybrid approach whereas the others use a reactive approach. All but SDAR, AnonDSR, ARM, HANOR, MASK, and ZAP offer location anonymity. ODAR, ASR, AODPR, MASK, and ASC claim to offer sender, receiver, communications and location anonymity. Only HANOR offers group anonymity. The wireless protocol classification offers a high-level view of the state-of-the-art wireless anonymous networks and corresponding anonymity properties. The overall classification of sixteen wireless anonymous network protocols is shown in Figure 7. Also, nine hybrid anonymous protocols are classified in Figure 8.

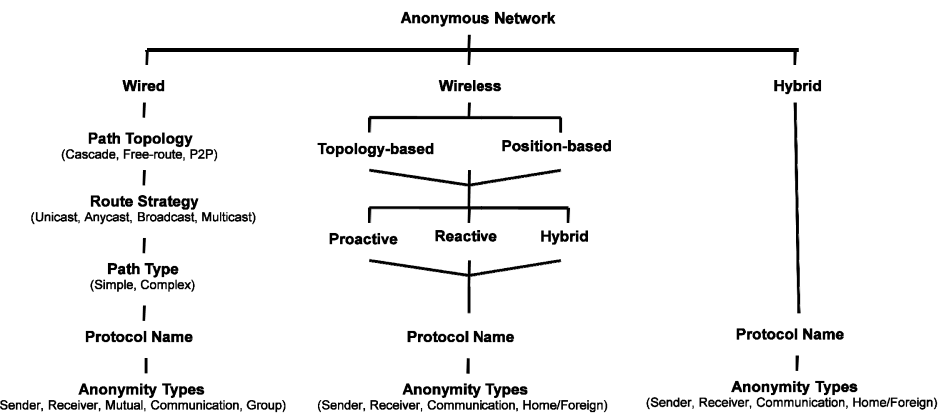

Figure 5: Tree Taxonomy with Anonymity Types

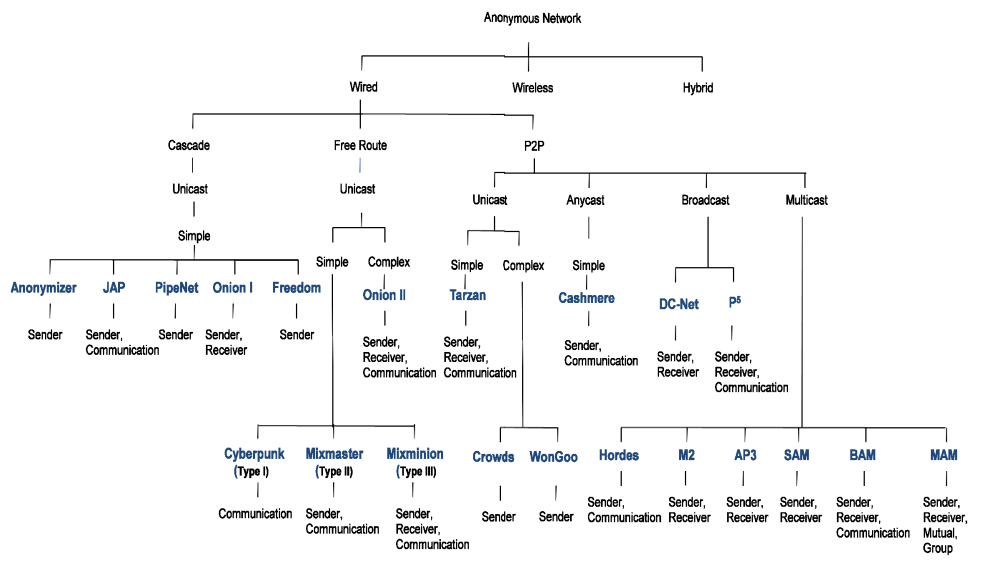

Figure 6: Classification of Wired Anonymous Networks

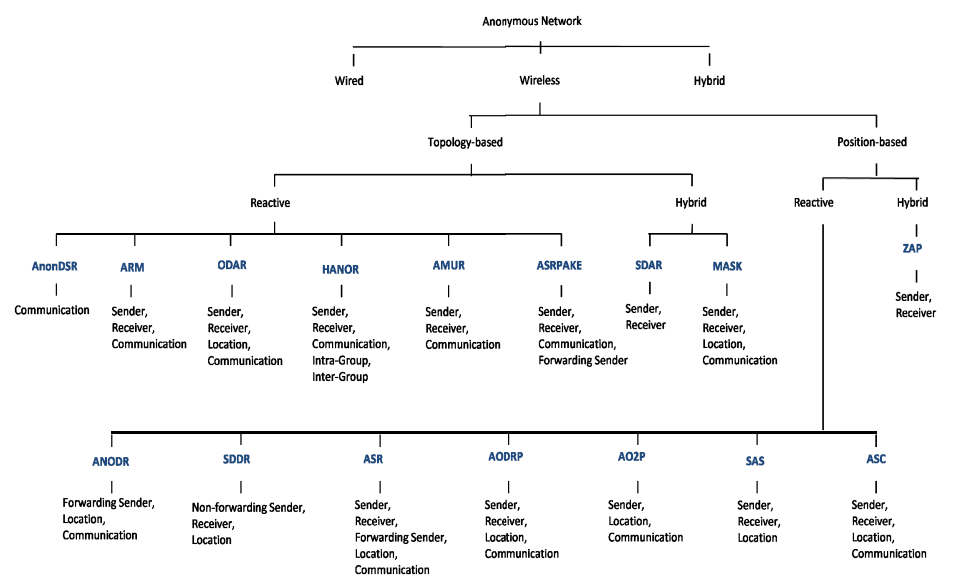

Figure 7: Classification of Wireless Anonymous Networks

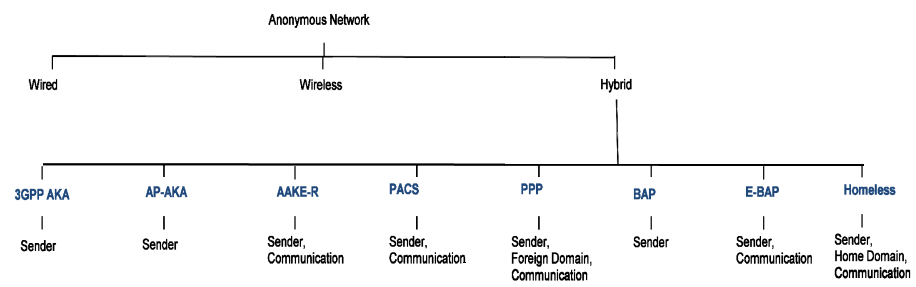

Figure 8: Classification of Hybrid Anonymous Networks 


\section{Taxonomy Advantages}

The primary advantages of these taxonomies include (1) opening up the possibility of easily identifying comparable anonymous system for further empirical or theoretical investigation and (2) identifying gaps in anonymous protocol design and development. For instance, out of the set of six P2P multicast anonymous protocols only M2, AP3, and SAM each offer sender and receiver anonymity. If an organization's evolving anonymity requirements and networking environment include anonymous multicast services, then the anonymity properties may be more formally proven [64] or protocols quantitatively compared with a suitable multicast metric [65]. Thus, an organization is better able to better defend itself against cyber attacks. Also, this classification revealed a gap in the security architecture for vehicular ad hoc networks (VANETs) as no known anonymous protocols for this subset of MANETs have been researched [66]. Thus, the taxonomy serves as an innovative guide for future researchers' anonymity interests.

\section{CONCLUSION}

This paper described an innovative CT to facilitate the systematic definition and comprehensive classification of anonymity of wired and wireless anonymous communications networks. The taxonomy considered seven desired anonymity properties, six assumed adversary capabilities, and fifteen special network types. An expanded cubic anonymity definition was expressed. The assumed adversary capabilities were described. The wired and wireless network types were further refined. Finally, the taxonomy was used to compare and contrast over forty-six state-of-the-art existing or proposed anonymous networks. Future work should more closely examine the last component more - adversary capability - to better articulate the overt and hidden adversary assumptions and implications for each anonymous protocol. Also, a more detailed classification of hybrid anonymous networks used for anonymous authentication and key exchange is in order.

\section{REFERENCES}

[1] J. Boyan, "The Anonymizer," in CMC Magazine, 1997.

[2] M. Reiter and A. Rubin, "Crowds: Anonymity for web transactions," ACM Transactions on Information and System Security, pp. 66-92, 1998.

[3] M. J. Freedman and R. Morris, "Tarzan: A Peer-to-Peer Anonymizing Network Layer " presented at 9th ACM Conference on Computer and Communications Security (CCS), Washington DC, USA, 2002.

[4] S. Goel, M. Robson, M. Polte, and E. Sirer, "Herbivore: A Scalable and Efficient Protocol for Anonymous Communication," Cornell University, 2003.

[5] G. Danezis, R. Dingleldine, and N. Mathewson, "Mixminion: Design of a Type III Anonymous Remailer Protocol," in IEEE Symposium on Security and Privacy (SP), 2003.

[6] I. Goldberg and A. Shostack, "Freedom Network 1.0 Architecture and Protocols," 1999.

[7] R. Sherwood, B. Bhattacharjee, and A. Srinivasan, "P5: A Protocol for Scalable Anonymous Communication " presented at Security and Privacy, 2002.

[8] R. Dingledine, N. Mathewson, and P. Syverson, "Tor: The Second Generation Onion Router," in 13th USENIX Security Symposium, 2004.

[9] K. Il-Khatib, L. Korba, R. Song, and G. Yee, "Secure Dynamic Distributed Routing Algorithm for Ad hoc Wireless Networks," presented at International Conference on Parallel Processing Workshops (ICPPW), 2003

[10] J. Kong and X. Hong, "ANODR: ANonymous On Demand Routing with Untraceable Routes for Mobile Adhoc Networks," presented at 4th ACM International Symposium on Mobile Ad-hoc Networking \& Computing (MobiHoc), Annapolis MD, USA, 2003.

[11] Z. Zhang, W. Liu, and Y. Fang, "MASK: Anonymous On-Demand Routing in Mobile Ad Hoc Networks," IEEE Transactions on Wireless Communications, vol. 5, pp. 2376-2385, 2006.

[12] H. Fathi, S. Shin, K. Kobara, and H. Imai, "Protocols for Authenticated Anonymous Communications," in 18th International Symposium on Personal, Indoor and Mobile Radio Communications (PIMRC). Athens, Greece: IEEE, 2007.

[13] D. Samfat, R. Molva, and N. Asokan, "Untraceability in Mobile Networks," ACM MOBICOM, pp. 26-36, 1995.

[14] M. Zhang and Y. Fang, "Security Analysis and Enhancements of 3GPP Authentication and Key Agreement Protocol," IEEE Transactions on Wireless Communications, vol. 4, pp. 734- 742, 2005.

[15] V. Varadharajan and Y. Mu, "Preserving Privacy in Mobile Communications: a Hybrid Method," in International Conference on Personal Wireless Communciations: IEEE, 1997.

[16] G. Ateniese, A. Herzberg, H. Krawczyk, and G. Tsudik, "Untraceable Mobility or How to Travel Incognito," Computer Networks, vol. 31, pp. 871-884, 1999.

[17] Y. G., D. Wong, and X. Deng, "Anonymous and Authenticated Key Exchange for Roaming Networks," IEEE Transactions on Wireless Communications, vol. 6, pp. 3461-3472, 2007.

[18] S. Misra and G. Xue, "SAS: A Simple Anonymity Scheme for Clustered Wireless Sensor Networks," presented at IEEE International Conference on Communications (ICC), 2006.

[19] L. Xiao, X. Liu, G. W., D. Xuan, and Y. Liu, "A Design of Overlay Anonymous Multicast Protocol," presented at Parallel and Distributed Processing Symposium (IPDPS), 2006.

[20] J. Wang, C. Niu, and R. Shen, "Bus-based Anonymous Mulitcast in Peer-to-Peer Overlay," in International Conference on Network and Parallel Computing - Workshops. Dalian, China, 2007.

[21] J. C. Kao and R. Marculescu, "Real-Time Anonymous Routing for Mobile Ad Hoc Networks," presented at IEEE International Conference on Local Computer Networks, Tampa FL, USA, 2007.

[22] D. Kesdogan, "Evaluation of anonymity providing techniques using queuing theory," presented at 26th Annual IEEE Conference on Local Computer Networks (LCN) Tampa FL, USA, 2001.

[23] Y. Guan, X. Fu, R. Bettati, and W. Zhao, "An Optimal Strategy for Anonymous Communication Protocols," presented at 22nd International Conference on Distributed Computing Systems, College Station, TX, 2002.

[24] J. Liu, J. Kong, X. Hong, and M. Gerla, "Performance Evaluation of Anonymous Routing Protocols in MANETs," University of Alabama, 2005, pp. 6.

[25] J. Kong, J. Liu, X. Hong, D. Wu, and M. Gerla, On Performance Cost of On-demand Anonymous Routing Protocols in Mobile Ad Hoc Networks Springer US, 2007.

[26] J. S. Valacich, A. R. Dennis, L. M. Jessup, and J. F. Nunamaker, Jr., "A Conceptual Framework of Anonymity in Group Support Systems," presented at 25th Hawaii International Conference on System Sciences Kauai HI, USA, 1992.

[27] V. Sunderam, J. Pascoe, and R. Loader, "Towards a Framework for Collaborative Peer Groups," presented at 3rd IEEE/ACM International Symposium on Cluster Computing and the Grid (CCGrid) 2003.

[28] H. Tillwick and M. Olivier, "Towards a Framework for Connection Anonymity," presented at SAICSIT, 2005.

[29] B. Gedik and L. Ling, "Location Privacy in Mobile Systems: A Personalized Anonymization Model," 25th IEEE International Conference on Distributed Computing Systems (ICDCS), pp. 620$629,2005$.

[30] B. Gedik and L. Liu, "Protecting Location Privacy with Personalized k-Anonymity: Architecture and Algorithms," IEEE Transactions on Mobile Computing, vol. 6, 2007.

[31] C. Bowen and T. Martin, "A Survey of Location Privacy and an Approach for Solitary Users," presented at 40th Annual Hawaii International Conference on System Sciences Hawaii, 2007. 
[32] A. Jones, "Anonymous Communication on the Internet," Indiana Unversity, 2004, pp. 13.

[33] A. E. Pascual, "Anonymous and Untraceable Communications," 21 June 2000.

[34] K. Sampigethaya and R. Poovendran, "A Survey on Mix Networks and Their Secure Applications," IEEE, vol. 94, pp. 2142-2181, 2006.

[35] J. Liu, X. Hong, J. Kong, Q. Zheng, N. Hu, and P. Bradford, "A Hierarchical Anonymous Routing Scheme for Mobile Ad-Hoc Networks," presented at Military Communications Conference (MILCOM), 2006.

[36] T. Lu, B. Fang, Y. Sun, and X. Cheng, "WonGoo: A Peer-to-Peer Protocol for Anonymous Communication," Journal of Parallel and Distributed Processing Techniques and Applications, vol. 3, pp. 1102-1106 2004.

[37] R. Song, L. Korba, and G. Yee, "AnonDSR: Efficient Anonymous Dynamic Source Routing for Mobile Ad-hoc Networks," presented at 3rd ACM Workshop on Security of Ad hoc and Sensor Networks Alexandria VA, USA 2005.

[38] A. Boukerche, K. El-Khatib, L. Xu, and L. Korba, "SDAR: a secure distributed anonymous routing protocol for wireless and mobile ad hoc networks," presented at 29th Annual IEEE International Conference on Local Computer Networks, 2004.

[39] A. Pfitzmann and M. Kohntopp, "Anonymity, Unobservability, and Pseudonymity - A Proposal for Terminology," presented at Workshop on Design Issues in Anonymity and Unobservability, Berkeley, CA, USA, 2000.

[40] D. L. Chaum, "The Dining Cryptographers Problem: Unconditional Sender and Recipient Untraceability," Journal of Cryptology, vol. 1, pp. 65-75, 1988.

[41] A. Pfitzmann and M. Waidner, "Networks without User Observability - Design Options," presented at Workshop on the Theory and Application of Cryptographic Techniques, Linz, Austria, 1986.

[42] M. Waidner, "Unconditional Sender and Recipient Untraceability in Spite of Active Attacks," presented at Workshop on the Theory and Application of Cryptographic Techniques on Advances in Cryptology, Houthalen, Belgium 1990.

[43] D. A. Cooper and K. P. Birman, "Preserving Privacy in a Network of Mobile Computers," presented at IEEE Symposium on Security and Privacy 1995.

[44] B. Zhu, Z. Wna, M. S. Kankanhalli, F. Bao, and R. H. Deng, "Anonymous Secure Routing in Mobile Ad-Hoc Networks," 29th Annual IEEE International Conference on Local Computer Networks pp. 102-108, 2004.

[45] M. Gruteser and D. Grunwald, "Anonymous Usage of LocationBased Services Through Spatial and Temporal Cloaking," presented at Proceedings of MobiSys 2003: The 1st International Conference on Mobile Systems, Applications, and Services, San Francisco, CA, 2003.

[46] G. Ghinita, P. Kalnis, and S. Skiadopoulos, "PRIVE: Anonymous Location-Based Queries in Distributed Mobile Systems," Department of Computer Science, National University of Singapore, Singapore 2006.

[47] A. R. Beresford and F. Stajano, "Location Privacy in Pervasive Computing," IEEE Pervasive Computing, vol. 2, pp. 46-55, 2003.

[48] D. Sy, R. Chen, and L. Bao, "ODAR: On-Demand Anonymous Routing in Ad Hoc Networks," University of California, Irvine, 2006, pp. 1-10.

[49] X. Wu and B. Bhargava, "AO2P: Ad Hoc On-Demand PositionBased Private Routing Protocol," IEEE Transactions on Mobile Computing, vol. 4, pp. 335-348, 2005.

[50] D. L. Chaum, "Untraceable Electronic Mail, Return Addresses, and Digital Pseudonyms," Communications of the ACM, vol. 24, pp. 84-88, 1981.

[51] B. Levine and C. Shields, "Hordes: A Multicast-based Protocol for Anonymity," Journal of Computer Security, vol. 10, pp. 213-240, 2002.

[52] L. Zhuang, F. Zhou, B. Zhao, and A. Rowstron, "Cashmere: Resilient Anonymous Routing," presented at 2nd Symposium on Networked Systems Design \& Implementation (NSDI), Boston MA, 2005.

[53] M. K. Reiter and A. D. Rubin, "Crowds: Anonymity for Web Transactions," ACM Transactions on Information and System Security, vol. 1, pp. 66-92, 1998.
N. A. Fraser, "Mitigiating Distributed Denial of Service Attacks in an Anonymous Routing Environment: Client Puzzles and Tor," in Computer and Electrical Engineering, vol. Master. WrightPatterson Air Force Base: Air Force Institute of Technology, 2006, pp. 122.

[55] X. Wu and E. Bertino, "Achieving K-anonymity in Mobile Ad Hoc Networks," presented at 1st IEEE ICNP Workshop on Secure Network Protocols (NPSec). , 2005.

[56] T. Eggendorfer, "Anonymous Surfing with Java Anonymous Proxy," in $W W W$. LINUX-MAGAZINE.COM, 2005, pp. 44-46.

[57] M. G. Reed, P. F. Syverson, and D. M. Goldschlag, "Anonymous Connections and Onion Routing," IEEE Journal on Selected Areas in Communications, vol. 16, 1998.

[58] W. Dai, "PipeNet 1.1," 1998.

[59] L. Cottrell, "Mixmaster Protocol Version 3," Anonymizer Inc., 2001.

[60] G. Perng, M. Reiter, and C. Wang, "M2: Multicasting Mixes for Efficient and Anonymous Communication," presented at 26th IEEE International Conference on Distributed Computing Systems, 2006.

[61] A. Mislove, G. Oberoi, A. Post, C. Reis, P. Druschel, and D. Wallach, "AP3: Cooperative, Decentralized Anonymous Communication," in 11th ACM SIGOPS European Workshop. Leuven, Belgium, 2004.

[62] S. Seys and B. Preneel, "ARM: Anonymous Routing Protocol for Mobile Ad hoc Networks," presented at 20th International Conference on Advanced Information Networking and Applications (AINA), Vienna, AU, 2006.

[63] M. Rahman, M. Mambo, A. Inomata, and E. Okamoto, "An Anonymous On-Demand Position-Based Routing in Mobile Ad Hoc Networks," presented at International Symposium on Applications and the Internet (SAINT) 2006.

[64] D. Kelly, R. Raines, R. Baldwin, B. Mullins, and M. Grimaila, "Towards Mathematically Modeling the Anonymity Reasoning Ability of an Adversary," presented at IPCCC International Workshop on Information and Data Assurance, Austin TX, USA, 2008.

[65] D. Kelly, R. Raines, R. Baldwin, B. Mullins, and M. Grimaila, "A Survey of the State of the Art in Anonymity Metrics," in 1st Workshop on Network Data Anonymization (NDA 2008): ACM, 2008.

[66] K. PloBl, T. Nowey, and C. Mletzko, "Towards a Security Architecture for Vehicular Ad Hoc Networks," presented at First International Conference on Availability, Reliability and Security (ARES), 2006. 